\title{
Primordial lepton oscillations and baryogenesis
}

\author{
Yuta Hamada ${ }^{a, b}$ and Ryuichiro Kitano ${ }^{a, c}$ \\ a Theory Center, High Energy Accelerator Research Organization (KEK), \\ Tsukuba 305-0801, Japan \\ ${ }^{b}$ Department of Physics, University of Wisconsin, \\ Madison, WI 53706, U.S.A. \\ ${ }^{c}$ Department of Particle and Nuclear Physics, \\ The Graduate University for Advanced Studies (Sokendai), \\ Tsukuba 305-0801, Japan \\ E-mail: yhamada@wisc.edu, ryuichiro.kitano@kek.jp
}

ABSTRACT: The baryon asymmetry of the Universe should have been produced after the inflation era. We consider the possibility that the asymmetry is generated by the flavor oscillations in the reheating process after inflation, so that the baryon asymmetry is realized already at the beginning of the radiation dominated era. In the seesaw model, we show that the propagators of the left-handed leptons generically have flavor mixings in the thermal background, that can generate flavor-dependent lepton asymmetry through the $C P$ violation in the oscillation phenomena. The flavor dependent rates for the wash-out process can leave the net asymmetry today.

KEYwords: Cosmology of Theories beyond the SM, CP violation, Neutrino Physics

ARXIV EPRINT: 1609.05028 


\section{Contents}

1 Introduction 1

2 Lepton propagators in the early Universe 2

3 Baryon asymmetry 4

$\begin{array}{llr}4 & \text { Conclusion } & 6\end{array}$

\section{Introduction}

The $C P$ violation in the neutrino oscillation can produce flavor dependent particleantiparticle asymmetry. Although it has not been established experimentally, the discovery of such phenomena will be a quite important hint for the mystery of the baryon asymmetry of the Universe. The mechanism for the baryon asymmetry before the electroweak phase transition requires generation of primordial $B-L$ asymmetry, rather than $B$ asymmetry, due to the $B+L$ breaking electroweak sphaleron process [1-4]. Within the field content of the Standard Model, the Majorana neutrino mass term, $l l H H$, is the lowest dimensional operator which breaks $B-L$ explicitly. Therefore, it is quite reasonable that the $C P$ violation in the neutrino interactions is responsible for baryogenesis.

The standard leptogenesis scenario [5] assumes that the $C P$ violation in the decays of right-handed neutrinos produces the lepton asymmetry, and thus it requires the production of the right-handed neutrinos after the inflation and their decays when the $L$-violating interactions gets sufficiently ineffective. It has been extensively studied such possibilities and viable parameter regions are investigated. It has been found that the mechanism works for high enough reheating temperatures. For the review, see refs. $[6,7]$ for example.

There is another interesting period of time where the out-of-equilibrium condition is satisfied automatically. After the inflation, the decays of the inflaton can reheat the Universe, producing the radiation energy which eventually dominates the Universe. In the course of reheating, high-energy particles are produced by the decay process, and each particles lose their energy by scattering with thermal plasma. This energy-loss process is obviously a one-way process, and thus provides us with perfect environment for baryogenesis. In refs. $[10,11]$, such a possibility has been studied where the scattering of the leptons during the reheating process produces the baryon asymmetry of the Universe.

In this paper, we consider the oscillation phenomena of the left-handed leptons (neutrinos and charged leptons) in the thermal background and the possibility to produce lepton asymmetry via its $C P$ violation. ${ }^{1}$ The inflaton decays into leptons in a particular combination of the flavor eigenstates. Since the lepton propagators in the thermal background are

\footnotetext{
${ }^{1}$ See refs. $[8,9]$ for baryogenesis from the oscillations of left-handed leptons which are originated from the decay of right-handed neutrinos.
} 
not diagonal in the flavor basis, the leptons can change the flavor during the propagation. The $C P$ violation in the oscillation, i.e., the indirect $C P$ violation in the inflaton decays, can produce the flavor dependent lepton asymmetry although no net lepton asymmetry is generated. The flavor dependent lepton asymmetry, in turn, can be converted into the net asymmetry by the flavor dependent wash-out process due to, for example, the $l l H H$ interactions. We find that the baryon asymmetry of the Universe can be explained by this mechanism, even in the case where the reheating temperature is much lower than the masses of the right-handed neutrinos and there is no decays of the inflatons into righthanded neutrinos, which, therefore, have never shown up in the history of the Universe.

This paper is organized as follows. In section 2, we discuss the lepton oscillation phenomena in the context of the seesaw model. We show that observed baryon asymmetry of the Universe can be explained by the primordial lepton oscillation in section 3. Section 4 is devoted for summary and conclusion.

\section{Lepton propagators in the early Universe}

In this section, we first calculate the propagators of the left-handed leptons in the thermal background, and show that the thermal correction to the dispersion relation causes the flavor oscillations as in the case of the neutrino oscillation in the vacuum by the mass differences. As a concrete scenario, we consider the seesaw model [12-16] in which the left-handed leptons have flavor off-diagonal interactions.

The Lagrangian is given by

$$
\begin{aligned}
\mathcal{L}_{\text {int }}= & -y_{\nu}^{i j} \bar{N}_{i} P_{L}\left(l_{j} \cdot \tilde{H}\right)+\text { h.c. } \\
& +\frac{M_{i}}{2} \bar{N}_{i}^{c} N_{i}+\text { h.c. } \\
& +y_{e}^{l} \bar{e}_{l} P_{L}\left(l_{l} \cdot H\right)+\text { h.c. }
\end{aligned}
$$

We have diagonalized the charged lepton Yukawa coupling $y_{e}$, that defines the flavor eigenstate. The right-handed neutrinos, $N_{i}$, are introduced and $M_{i}$ are their Majorana masses.

In a high temperature medium where the Higgs particles are in the thermal bath, the Yukawa interactions in eq. (2.1) affect the propagators of the $l_{i}$ fields. Following ref. [17], the propagator of a fermion field in the momentum space is parametrized as

$$
S(K)=[(1+a) \not K+b \psi]^{-1},
$$

where $K^{\mu}=(\omega, \mathbf{k})$ is the four momentum and $u^{\mu}=(1,0,0,0)$. The coefficients, $a$ and $b$, are functions of $\omega$ and $k=|\mathbf{k}|$. By looking at the pole of the propagator, the dispersion relation is modified to

$$
\omega=k-\operatorname{Re}\left[\frac{b}{1+a}\right] .
$$

The non-vanishing functions, $a$ and $b$, are the effects of the interactions. At the leading order in the perturbation theory, one can ignore $a$. The real part of the function $b$ is calculated to be

$$
b_{l l^{\prime}} \simeq-\frac{T^{2}}{16 k}\left(y_{e}^{l}\right)^{2} \delta_{l l^{\prime}}+\frac{\pi^{2} T^{4}}{9 M_{k}^{4}} y_{\nu}^{k l *} y_{\nu}^{k l^{\prime}} k
$$


for $\omega \sim k \gg T$, where $T$ is the temperature. We have assumed here that $T k \ll M_{i}^{2}$. For $T k \gtrsim M_{i}^{2}$, one obtains the term similar to the first term which stems from the charged lepton Yukawa interactions. The second term provides flavor non-diagonal entries, responsible for the oscillation. The second term is not quite the "thermal mass," since the dispersion relation is still $\omega=0$ in the $k \rightarrow 0$ limit. However, it does modify the dispersion relation as in eq. (2.3), and causes the flavor oscillation phenomena as we discuss below. The contribution from the gauge interactions are flavor universal, and thus can be ignored for our purpose.

The second term is suppressed by $M_{i}^{4}$, which can be understood by the operator analyses. For $M_{i} \gg T$, one can integrate out the $N_{i}$ fields, and effective interaction terms with dimension five or higher are generated. The odd-dimensional operators, such as $l l H H$ and $l l H \partial^{2} H$, break the $L$ number as well as the Higgs number, and thus would not cause the forward elastic scattering to modify the propagators. The dimension-six operators, $\bar{l} \gamma^{\mu} l\left(i H^{\dagger} \partial_{\mu} H+\right.$ h.c. $)$, would not contribute as long as there is no chemical potential for the Higgs fields. Therefore, the first contribution appears from the dimension-eight operators which are suppressed by $M_{i}^{4}$. Nevertheless, for a large enough $k$,

$$
k^{2} \geq \frac{9}{16 \pi^{2}} \frac{\left(y_{e}^{l}\right)^{2}}{y_{\nu}^{k l *} y_{\nu}^{k l^{\prime}}} \frac{M_{k}^{2}}{T^{2}} M_{k}^{2}
$$

the second term in eq. (2.4) dominates over the first one.

The matrix $b$ can be diagonalized by a unitary transformation:

$$
U^{\dagger} b U=b^{\text {diag }} .
$$

The unitary matrix $U$ is different from the PMNS matrix [18, 19], and the actual form cannot be determined only from the low energy data. The neutrino oscillation can be understood in the standard quantum mechanical considerations. In the basis of diagonal $b$, the neutrino wave function is given by $[20]$

$$
|t=\Delta t\rangle=e^{-i \omega_{i} \Delta t} e^{i k_{i} \Delta x}|t=0\rangle
$$

and the differences of the dispersion relations appear in the phase of the interference terms in the transition rates of $l_{l} \rightarrow l_{l^{\prime}}$ :

$$
\begin{aligned}
\delta \phi_{j k} & =\left(\omega_{j}-\omega_{k}\right) \Delta t-\left(k_{j}-k_{k}\right) \Delta x \\
& =\left(k_{j}-k_{k}\right)(\Delta t-\Delta x)-\Delta b_{j k} \Delta t \\
& \simeq-\Delta b_{j k} \Delta t,
\end{aligned}
$$

where $\Delta b_{j k}=b_{j}^{\text {diag }}-b_{k}^{\text {diag }}$, and $\Delta t$ and $\Delta x$ are time and length of the travel, respectively. The time $\Delta t$ should be taken as the mean free time of the lepton propagation, $\Delta t \simeq \Gamma^{-1}$, after which the leptons lose their momenta or pair annihilate with the leptons in the thermal bath. The dominant scattering process is through the $\mathrm{SU}(2)$ gauge interactions which give a flavor universal $\Gamma$ :

$$
\Gamma \sim \frac{g_{2}^{2}}{4 \pi} T
$$


Essentially the same result can be derived from field theory [21]. In the basis of diagonal $b$, the denominator of the propagators is

$$
\frac{1}{\left(\omega+b_{i}^{\text {diag }}\right)^{2}-k^{2}+i \omega \Gamma},
$$

where $\Gamma$ is given in eq. (2.9). The field theoretic computation of the probability involves the phase space integration over the propagators:

$$
\int_{0}^{\infty} d \omega \frac{1}{\left(\omega+b_{j}\right)^{2}-k^{2}+i \omega \Gamma} \frac{1}{\left(\omega+b_{k}\right)^{2}-k^{2}-i \omega \Gamma} \simeq \frac{\pi}{k^{2} \Gamma}\left[\frac{1}{1+2 i \Delta b_{j k} / \Gamma}\right] .
$$

We see the oscillation effect $1 /\left(1+2 i \Delta b_{j k} / \Gamma\right)$, which is similar to the quantum mechanical consideration, $\exp \left(-i \Delta b_{j k} / \Gamma\right)$, for $\Delta b_{j k} / \Gamma \ll 1$.

\section{Baryon asymmetry}

Now we consider the possible scenario for realizing the baryon asymmetry through the primordial oscillation phenomena.

We assume that the high energy leptons are generated by the decays of the inflaton $\phi$ :

$$
\phi \rightarrow l_{i}+X
$$

and its $C P$ conjugate process. The part of the final state $X$ is arbitrary, and the decay mode above is not even necessary to be the dominant one. For example, $X=H+e_{j}^{c}$ makes $\phi$ gauge singlet. We also assume that the reheating temperature of the Universe, $T_{R}$, is lower than $10^{12} \mathrm{GeV}$ so that the Yukawa interaction of the tau leptons is in the thermal equilibrium [22]. In this circumstance, one can distinguish lepton asymmetries in $l_{\tau}$ and $l_{e, \mu}$ as in the case of flavored leptogenesis [23-26], where indices are defined in the basis where the charged lepton Yukawa matrix is diagonal.

As we have seen in the previous section, the neutrino Yukawa interaction is not diagonal in this basis. The leptons obtain the off-diagonal components in their propagator in the thermal plasma as in eq. (2.4). Therefore, the leptons generated through the inflaton decays undergo the flavor oscillations until the first scattering where the leptons lose their energy.

In the basis where the propagator is diagonalized, the flavor eigenstate is expressed as

$$
|l(e, \mu, \tau)\rangle=\sum_{j=1,2,3} U_{j l}^{\dagger}|j\rangle
$$

and the inflaton decays provide lepton states which are a linear combination of the eigenstates of the Hamiltonian:

$$
\left|l_{\phi}\right\rangle=\sum_{j=1,2,3} V_{j}^{*}|j\rangle .
$$

Here, $U$ is a unitary matrix same as that in eq. (2.6), whereas $V$ is a normalized vector. By combining eqs. (2.8), (3.2), and (3.3), the $C P$ asymmetry is given by the oscillation formula [20, 27]:

$$
P_{l}-P_{\bar{l}}=4 \sum_{j>k} \operatorname{Im}\left(U_{l j} V_{j}^{*} V_{k} U_{l k}^{*}\right) \int d k f(k) \sin \frac{\Delta b_{j k}(k)}{\Gamma} .
$$


Here $P_{l(\bar{l})}$ represents the oscillation probability of $l_{\phi} \rightarrow l_{l}\left(\bar{l}_{\phi} \rightarrow \bar{l}_{l}\right), f(k)$ is the momentum distribution of neutrinos from inflaton decay with $\int d k f(k)=1$, and we have evaluated asymmetry at the stage of the first scattering, that is, $\Delta t \simeq \Gamma^{-1}$. In the following, we take $V_{1}=0$ for simplicity. Then, the $C P$ asymmetry is

$$
\begin{aligned}
P_{l}-P_{l} & =4 \operatorname{Im}\left(U_{l 3} V_{3}^{*} V_{2} U_{l 2}^{*}\right) \int d k f(k) \sin \frac{\Delta b_{32}(k)}{\Gamma} \\
& \simeq 4 \operatorname{Im}\left(U_{l 3} V_{3}^{*} V_{2} U_{l 2}^{*}\right) \frac{\Delta b_{32}\left(m_{\phi}\right)}{\Gamma} .
\end{aligned}
$$

In the second line, we have expanded the sine function, and used the relation that the expectation value of momentum, $\int d k k f(k)$, is the order of $m_{\phi}$ which is the mass of the inflaton. Eq. (3.5) is generally non-vanishing and the $C P$-asymmetry factor, $A_{C P}=$ $\operatorname{Im}\left(U_{l 3} V_{3}^{*} V_{2} U_{l 2}^{*}\right)$, can easily be of order unity. Since the asymmetry vanishes when we take the sum over the $l$ index, there is no net lepton asymmetry generated. The tau asymmetry is compensated by the $e$ and $\mu$ asymmetry.

At the stage of the first scattering, however, the flavor eigenstate is not a meaningful concept since the time scale of the first scattering is much faster than the scattering through the Yukawa interaction for the charged tau lepton. This means that the density matrix of the lepton number is not diagonal in the flavor basis. The flavor dependent asymmetry becomes physical later at the time scale of the scattering through the tau Yukawa interaction, where the off-diagonal components of the density matrix dump to zero while the diagonal components, which we calculated, are left time-independent [23-26]. Note that, if the interaction rate of neutrino Yukawa couplings is larger than that of tau Yukawa, it could generate the off-diagonal components of the density matrix, which threatens our baryogenesis mechanism. As long as right-handed neutrinos are heavy, it is safe because we know that the dominant interaction rate through the $l l H H$ operator is smaller than Hubble rate as we discuss later. ${ }^{2}$

Since we assume that the temperature is low enough that the tau Yukawa interaction is in the thermal equilibrium, one can identify the tau asymmetry, and thus the generated flavor dependent asymmetry can be separately treated. In particular, the $\Delta L=2$ process through the $l l H H$ interaction can wash out the lepton asymmetry in a flavor dependent manner, that results in the generation of the net lepton asymmetry. If we assume the hierarchical neutrino masses in normal hierarchy, one can consider the situation where only the $l_{3} l_{3} H H$ interaction is effective. The interaction rate for the $\Delta L=2$ process is approximately given by

$$
\Gamma_{l}^{\mathrm{w} . \mathrm{o}} \simeq \frac{\left|U_{l 3}^{\mathrm{PMNS}}\right|^{2}}{\pi^{2}} \frac{m_{\nu 3}^{2}}{v^{4}} T_{R}^{3}
$$

where $U^{\text {PMNS }}$ is the PMNS matrix, $m_{\nu i}$ is the neutrino mass, and $v \simeq 246 \mathrm{GeV}$. Compared with the expansion rate of the Universe, we obtain

$$
\frac{\Gamma_{l}^{\mathrm{w} . \mathrm{o}}}{H}=\frac{T}{10^{12} \mathrm{GeV}} \times\left\{\begin{array}{l}
0.03, l=\tau \\
0.02, l=\mu+e
\end{array}\right.
$$

\footnotetext{
${ }^{2}$ We thank Sacha Davidson for discussion on the flavor basis and the time scales of the various interactions.
} 
Here we take the observed values of mixing angles and $m_{\nu 3} \simeq 0.05 \mathrm{eV}[20]$. Note that this quantity does not depend on the Dirac or Majorana phases.

As a result, we obtain the net lepton asymmetry as

$$
\begin{aligned}
\frac{n_{L}}{s} & \sim \frac{n_{\phi}}{s} \times B_{\phi \rightarrow l} \times A_{C P} \times \frac{\Delta b_{23}}{\Gamma} \times\left(\frac{\Gamma_{\tau}^{\text {w.o. }}}{H}-\frac{\Gamma_{\mu+e}^{\text {w.o. }}}{H}\right) \\
& \sim \frac{n_{\phi}}{s} \times B_{\phi \rightarrow l} \times A_{C P} \times \frac{\Delta b_{23}}{\Gamma} \times 0.01 \times\left(\frac{T_{R}}{10^{12} \mathrm{GeV}}\right),
\end{aligned}
$$

where $B_{\phi \rightarrow l}$ is the branching ratio of the inflaton decays into leptons. The first factor is the inflaton abundance, and the factor of the difference of the $\Gamma^{\text {w.o. }} / H$ describes the flavor-dependent wash-out effects. The number density of the inflaton is

$$
\frac{n_{\phi}}{s} \simeq \frac{T_{R}}{m_{\phi}}
$$

and the splitting of the dispersion relation is

$$
\frac{\Delta b_{23}}{\Gamma} \sim \frac{4 \pi^{3} m_{\phi} T_{R}^{3}}{9 g_{2}^{2} M_{k}^{4}}\left|y_{\nu}^{k 3}\right|^{2},
$$

by taking $k \sim m_{\phi}$. Therefore, putting altogether, we obtain the baryon asymmetry

$$
\frac{n_{B}}{s} \sim \frac{n_{L}}{s} \sim 10^{-7} \times B_{\phi \rightarrow l} \times A_{C P} \times\left(\frac{T_{R}}{10^{12} \mathrm{GeV}}\right)^{2}\left(\frac{10}{M / T_{R}}\right)^{-3},
$$

where $M$ is the right-handed neutrino mass which we take, for simplicity, to be common for all flavors. We see that the baryon asymmetry, $n_{B} / s \sim 10^{-10}$ [28], can be explained by this mechanism. It is interesting to note that the final formula is independent of the inflaton mass as long as $m_{\phi} \gg T_{R}$. We also note that eq. (2.5),

$$
k \geq \frac{3}{4 \pi} \frac{y_{e}^{l}}{\sqrt{y_{\nu}^{k l *} y_{\nu}^{k l}}} \frac{M}{T_{R}} M \sim 10^{12} \mathrm{GeV}\left(\frac{y_{e}^{l}}{10^{-2}}\right)\left(\frac{M}{10^{13} \mathrm{GeV}}\right)^{3 / 2} \sqrt{\frac{0.05 \mathrm{eV}}{m_{\nu 3}}}\left(\frac{10^{12} \mathrm{GeV}}{T_{R}}\right),
$$

can be satisfied for large $m_{\phi} \sim k$.

The estimate above is only valid when $M^{2} \gg T_{R} m_{\phi}$. For more general situations, the wash-out rates as well as the thermal correction to the propagators can be enhanced, and thus a larger asymmetry may be realized unless the neutrino Yukawa couplings are larger than tau Yukawa coupling. We leave such general analyses for future studies.

\section{Conclusion}

In the inflationary Universe, one of the natural possibilities to explain the baryon asymmetry is that the asymmetry is generated during the reheating era such that the radiation dominated Universe has started with non-vanishing asymmetry. Since the transition from the inflaton dominated era to the radiation dominated one is a one-way process, one can naturally satisfy the out-of-equilibrium condition for baryogenesis. Also, for high enough 
reheating temperatures, the $L$-violating interactions responsible for the neutrino masses and the electroweak sphaleron process is effective, that can provide the $B$-violating condition. The final condition is the $C P$ violation. In the reheating era, the natural location to look for the $C P$ violation is the decays of the inflaton. We indeed have shown that $C P$ violation in the flavor oscillation of the leptons can happen in the inflaton decays and that can explain the baryon asymmetry of the Universe.

\section{Acknowledgments}

We would like to thank Sacha Davidson for useful comments. This work is supported by JSPS KAKENHI Grant-in-Aid for Scientific Research (B) (No. 15H03669 [RK]), MEXT Grant-in-Aid for Scientific Research on Innovative Areas (No. 25105011 [RK]) and Grantin-Aid for JSPS Fellows (No. 16J06151 [YH]).

Open Access. This article is distributed under the terms of the Creative Commons Attribution License (CC-BY 4.0), which permits any use, distribution and reproduction in any medium, provided the original author(s) and source are credited.

\section{References}

[1] G. 't Hooft, Computation of the Quantum Effects Due to a Four-Dimensional Pseudoparticle, Phys. Rev. D 14 (1976) 3432 [Erratum ibid. D 18 (1978) 2199] [InSPIRE].

[2] N.S. Manton, Topology in the Weinberg-Salam Theory, Phys. Rev. D 28 (1983) 2019 [INSPIRE].

[3] F.R. Klinkhamer and N.S. Manton, A Saddle Point Solution in the Weinberg-Salam Theory, Phys. Rev. D 30 (1984) 2212 [INSPIRE].

[4] V.A. Kuzmin, V.A. Rubakov and M.E. Shaposhnikov, On the Anomalous Electroweak Baryon Number Nonconservation in the Early Universe, Phys. Lett. B 155 (1985) 36 [INSPIRE].

[5] M. Fukugita and T. Yanagida, Baryogenesis Without Grand Unification, Phys. Lett. B 174 (1986) 45 [InSPIRE].

[6] W. Buchmüller, R.D. Peccei and T. Yanagida, Leptogenesis as the origin of matter, Ann. Rev. Nucl. Part. Sci. 55 (2005) 311 [hep-ph/0502169] [INSPIRE].

[7] S. Davidson, E. Nardi and Y. Nir, Leptogenesis, Phys. Rept. 466 (2008) 105 [arXiv:0802.2962] [INSPIRE].

[8] B. Garbrecht, Baryogenesis from Mixing of Lepton Doublets, Nucl. Phys. B 868 (2013) 557 [arXiv: 1210.0553] [INSPIRE].

[9] B. Garbrecht and I. Izaguirre, Phenomenology of Baryogenesis from Lepton-Doublet Mixing, Nucl. Phys. B 896 (2015) 412 [arXiv:1411.2834] [INSPIRE].

[10] Y. Hamada and K. Kawana, Minimal leptogenesis, arXiv:1510.05186 [INSPIRE].

[11] Y. Hamada, K. Tsumura and D. Yasuhara, The reheating era leptogenesis in models with seesaw mechanism, arXiv: 1608.05256 [INSPIRE]. 
[12] P. Minkowski, $\mu \rightarrow$ er at a Rate of One Out of $10^{9}$ Muon Decays?, Phys. Lett. B 67 (1977) 421 [inSPIRE].

[13] T. Yanagida, Horizontal Symmetry And Masses Of Neutrinos, in proceedings of Workshop on Unified Theory and Baryon Number of the Universe, O. Sawada and A. Sugamoto eds., KEK, Tsukuba (1979) [INSPIRE].

[14] M. Gell-Mann, P. Ramond and R. Slansky, Complex Spinors and Unified Theories, Conf. Proc. C 790927 (1979) 315 [arXiv: 1306.4669] [InSPIRE].

[15] S.L. Glashow, The Future of Elementary Particle Physics, NATO Sci. Ser. B 61 (1980) 687 [INSPIRE].

[16] R.N. Mohapatra and G. Senjanović, Neutrino Mass and Spontaneous Parity Violation, Phys. Rev. Lett. 44 (1980) 912 [INSPIRE].

[17] H.A. Weldon, Effective Fermion Masses of Order gT in High Temperature Gauge Theories with Exact Chiral Invariance, Phys. Rev. D 26 (1982) 2789 [INSPIRE].

[18] B. Pontecorvo, Inverse beta processes and nonconservation of lepton charge, Sov. Phys. JETP 7 (1958) 172 [INSPIRE].

[19] Z. Maki, M. Nakagawa and S. Sakata, Remarks on the unified model of elementary particles, Prog. Theor. Phys. 28 (1962) 870 [inSPIRE].

[20] Particle Data Group collaboration, K.A. Olive et al., Review of Particle Physics, Chin. Phys. C 38 (2014) 090001 [InSPIRE].

[21] N. Arkani-Hamed, J.L. Feng, L.J. Hall and H.-C. Cheng, CP violation from slepton oscillations at the LHC and NLC, Nucl. Phys. B 505 (1997) 3 [hep-ph/9704205] [InSPIRE].

[22] J.M. Cline, K. Kainulainen and K.A. Olive, Protecting the primordial baryon asymmetry from erasure by sphalerons, Phys. Rev. D 49 (1994) 6394 [hep-ph/9401208] [InSPIRE].

[23] A. Abada, S. Davidson, F.-X. Josse-Michaux, M. Losada and A. Riotto, Flavor issues in leptogenesis, JCAP 04 (2006) 004 [hep-ph/0601083] [INSPIRE].

[24] E. Nardi, Y. Nir, E. Roulet and J. Racker, The importance of flavor in leptogenesis, JHEP 01 (2006) 164 [hep-ph/0601084] [INSPIRE].

[25] A. Abada, S. Davidson, A. Ibarra, F.X. Josse-Michaux, M. Losada and A. Riotto, Flavour Matters in Leptogenesis, JHEP 09 (2006) 010 [hep-ph/0605281] [INSPIRE].

[26] P.S. Bhupal Dev, P. Millington, A. Pilaftsis and D. Teresi, Flavour Covariant Transport Equations: an Application to Resonant Leptogenesis, Nucl. Phys. B 886 (2014) 569 [arXiv: 1404.1003] [INSPIRE].

[27] P.I. Krastev and S.T. Petcov, Resonance Amplification and $t$ Violation Effects in Three Neutrino Oscillations in the Earth, Phys. Lett. B 205 (1988) 84 [INSPIRE].

[28] Planck collaboration, P.A.R. Ade et al., Planck 2015 results. XIII. Cosmological parameters, Astron. Astrophys. 594 (2016) A13 [arXiv:1502.01589] [INSPIRE]. 